\title{
Large volumes and spectroscopy of walking theories
}

\author{
L. Del Debbio, ${ }^{1}$ B. Lucini, ${ }^{2}$ A. Patella, ${ }^{3,4}$ C. Pica, ${ }^{5}$ and A. Rago ${ }^{4}$ \\ ${ }^{1}$ Higgs Centre for Theoretical Physics, School of Physics and Astronomy, University of Edinburgh, \\ Edinburgh EH9 3JZ, United Kingdom \\ ${ }^{2}$ College of Science, Swansea University, Singleton Park, Swansea SA2 8PP, United Kingdom \\ ${ }^{3}$ CERN-TH, CH-1211 Geneva, Switzerland \\ ${ }^{4}$ Centre for Mathematical Sciences, Plymouth University, Plymouth PL4 8AA, United Kingdom \\ ${ }^{5}$ CP-Origins \& Danish IAS, Campusvej 55, DK-5230 Odense M, Denmark
}

(Received 9 January 2016; published 11 March 2016)

\begin{abstract}
A detailed investigation of finite-size effects is performed for $\mathrm{SU}(2)$ gauge theory with two fermions in the adjoint representation, which previous lattice studies have shown to be inside the conformal window. The system is investigated with different spatial and temporal boundary conditions on lattices of various spatial and temporal extensions, for two values of the bare fermion mass representing a heavy and light fermion regime. Our study shows that the infinite-volume limit of masses and decay constants in the mesonic sector is reached only when the mass of the pseudoscalar particle $M_{\mathrm{PS}}$ and the spatial lattice size $L$ satisfy the relation $L M_{\mathrm{PS}} \geq 15$. This bound, which is at least a factor of three higher than what is observed in QCD, is a likely consequence of the different spectral signatures of the two theories, with the scalar isosinglet $\left(0^{++}\right.$glueball) being the lightest particle in our model. In addition to stressing the importance of simulating large lattice sizes, our analysis emphasizes the need to understand quantitatively the full spectrum of the theory rather than just the spectrum in the mesonic isotriplet sector. While for the lightest fermion measuring masses from gluonic operators proves to be still challenging, reliable results for glueball states are obtained at the largest fermion mass and, in the mesonic sector, for both fermion masses. As a byproduct of our investigation, we perform a finite-size scaling of the pseudoscalar mass and decay constant. The data presented in this work support the conformal behavior of this theory with an anomalous dimension $\gamma_{*} \simeq 0.37$.
\end{abstract}

DOI: 10.1103/PhysRevD.93.054505

\section{INTRODUCTION}

The recent discovery of the Higgs boson is the first step towards new physics beyond the Standard Model. As the properties of this new state are investigated in more detail, the constraints on models of new physics will become more stringent. It has been suggested in a long line of works stemming from Refs. [1-3] that at a more fundamental level the Higgs sector and the electroweak symmetry breaking mechanism of the Standard Model could be explained dynamically in terms of a novel strong interaction. In the light of recent developments, the study of scenarios of strongly interacting gauge theories beyond the Standard Model is playing an increasingly central role in both theoretical modeling and experimental searches. For instance, a preliminary observation of an excess in the diphoton decay channel has been recently reported by both ATLAS and CMS $[4,5]$. A strong interaction beyond the Standard Model can provide a natural interpretation of this excess. In the framework of a novel strong force, the

Published by the American Physical Society under the terms of the Creative Commons Attribution 3.0 License. Further distribution of this work must maintain attribution to the author(s) and the published article's title, journal citation, and DOI.
Higgs particle would be a composite state of the new interaction. The phenomenology of such a composite state is usually studied using effective theory descriptions, which rely on general (and possibly few) assumptions about the new dynamics. Nonperturbative effects due to the novel strong force are encoded in the low-energy constants of the effective Lagrangians. A naive guess for the candidate theory that describes this new force could be a gauge theory with matter coupled to the electroweak sector of the Standard Model. It has been known for a long time that the simplest model of this type, a rescaled version of QCD, would fail to satisfy the constraints from precision electroweak data. A more quantitative understanding of the nonperturbative dynamics of gauge theories is required in order to develop and test models that go beyond a naive rescaling of QCD. Depending on the gauge and matter content, these theories can be rather different from QCD. Computing from first principles their spectrum, and other low-energy constants relevant for phenomenology, would provide a most useful input for phenomenological studies. Unfortunately these quantities are notoriously difficult to compute for realistic candidate theories.

Lattice simulations with dynamical fermions have reached a stage where light fermion masses can be simulated effectively on large lattices using existing 
hardware. Therefore numerical methods provide an effective tool to investigate the phase diagram of gauge theories from first principles, and indeed several extensive studies have been performed in recent years, exploring the theory space by varying the number of colors and flavors, as well as the color representation of the fermionic matter.

Note that, in order to satisfy the constraints from electroweak precision data, and in particular to build a realistic flavor sector, the new theory is required to be near conformal, i.e. to have a multiscale dynamics, characterized by a clear separation between an IR and an UV scale. It is often advocated that such a separation be obtained by considering theories that are just outside the conformal window, or deformations of theories that have an infrared fixed point (IRFP), hence the "near-conformal" denomination. A large energy range where the Renormalisation Group (RG) evolution of the couplings is very slow (walking) is expected to appear, which leads to an approximate scale invariance at large distances. The low-energy dynamics is then characterized by the anomalous dimensions of the relevant operators at the IRFP, and large anomalous dimensions are typically required in order to construct phenomenologically viable models [6-11]. Lattice simulations yield quantitative results in strongly interacting theories from first principles. Holographic theories are an alternative way to perform explicit calculations using the perturbative dual description of the strong dynamics; several efforts to understand nearconformality in this framework have appeared over the years (see for instance [12-24]).

The main goal of our current numerical studies is the unambiguous identification of an IRFP, and the determination of the anomalous dimensions that characterize the dynamics. This is a difficult problem, since the systematic errors that affect lattice simulations need to be kept under control in order to highlight the interesting conformal behavior. First studies, which have been useful in the early stages of the numerical investigations, are affected by large systematics, which can obscure the physical features of these theories. Different approaches to conformality on the lattice have been suggested and tested over the years [25-40]. They include a variety of methods that range from the scaling of the spectrum to the explicit computation of the running of the couplings. Despite several large scale projects devoted to these problems [41-47], there is still some controversy in the literature, showing that great care must be exercised in the analysis of the lattice data.

In this work we focus on SU(2) gauge theory coupled to $n_{f}=2$ flavors of Dirac fermions in the adjoint representation of the gauge group. This model, introduced as a phenomenological extension of the Standard Model under the name of minimal walking technicolor (MWT) [48], is believed to have an IR fixed point $[26,27,31,35,49-52]$. We would like to find evidence for the conformal behavior from a study of the spectrum of this theory. The scaling of the spectrum, and the corrections to scaling, allow in principle a determination of the anomalous dimensions of the theory at the IR fixed point; see e.g. Refs. [32-34,53]. A precise determination of the spectrum would also allow a test of the Feynman-Hellmann relations that were proposed in Ref. [54]. The systematic errors on the spectrum due to the finite temporal and spatial extents of the lattice for MWT have been analyzed in a previous publication [55]: systematic errors of approximately $10 \%$ are common on lattices such that $L M_{\mathrm{PS}}<10$, where $M_{\mathrm{PS}}$ indicates the mass of the lightest pseudoscalar state in the spectrum. We have therefore embarked in large volume simulations of the theory, in order to provide results for the spectrum in a regime where systematic errors are below $1 \%$. The simulations have been performed using the Hi Rep code developed in Ref. [56]. A detailed description of the methodology, and of the lattices simulated in this work, is reported in Sec. II.

The new volumes simulated are large enough to avoid contamination from excited states effects, and to allow us to extrapolate the data for the spectrum to the infinite-volume limit for two values of the fermion mass. These are the first results for the spectrum of the MWT that can be extrapolated to the thermodynamical limit with an uncertainty at the percent level. The results for the full spectrum, including glueball states and the string tension, are discussed in Sec. III.

\section{METHODOLOGY}

We have simulated MWT using the RHMC algorithm described in Ref. [56]. In order to identify, and control, the systematic effects due to the finite size of the lattices, we have simulated the theory on a series of lattices, increasing both the temporal and the spatial extent of the system. The new runs are listed in Table I. All simulations have been performed at fixed lattice bare coupling $\beta=2.25$, and for two values of the fermion bare mass $a m_{0}=-1.05,-1.15$. As a further tool to investigate finite-volume effects, we have compared the spectrum obtained from simulations with the usual periodic boundary conditions to the one obtained with twisted boundary conditions, as defined in Ref. [57], and the one obtained with open boundary conditions, as described in Ref. [58]. The detailed implementation of the twisted and open boundary conditions is described below. In the infinite-volume limit, results should be independent of the boundary conditions, and therefore we can use the dependence on the boundary conditions to monitor whether or not the theory has reached the large volume asymptotic behavior. As discussed in Ref. [31], the smaller lattices A10 and A11 are in a regime where the distribution of the values of the Polyakov loop displays a double-peak structure. This regime where center symmetry is broken is usually not suitable for extracting the glueball spectrum [31]. The statistics for all lattices is targeted to a precise measurement of the mesonic spectrum, which is less demanding than the glueball analysis. The temporal 
TABLE I. List of lattices used in this study. The nomenclature for the lattices follows our earlier conventions. The lattices denoted by TW are the ones with twisted boundary conditions. The lattices denoted by $\mathrm{O}$ are the ones with open boundary conditions in one of the four Euclidean directions. The new runs are performed at two different values of the bare mass $a m_{0} . N_{\text {traj }}$ is the number of trajectories (of length $t_{\text {traj }}$ ) that we have generated. The value of the plaquette $\langle P\rangle$ and of the lowest eigenvalue of the Dirac operator $\lambda$ are also reported together with their respective integrated autocorrelation times $\tau_{P}$ and $\tau_{\lambda}$. All runs are performed at $\beta=2.25$.

\begin{tabular}{|c|c|c|c|c|c|c|c|c|}
\hline Lattice & $V$ & $-a m_{0}$ & $N_{\text {traj }}$ & $t_{\text {traj }}$ & $\langle P\rangle$ & $\tau_{P}$ & $\lambda$ & $\tau_{\lambda}$ \\
\hline A10 & $64 \times 8^{3}$ & 1.15 & 810 & 3 & $0.66536(22)$ & $3.6(1.2)$ & $0.2005(58)$ & $1.42(31)$ \\
\hline A11 & $64 \times 12^{3}$ & 1.15 & 530 & 1.5 & $0.66601(15)$ & $1.93(59)$ & $0.2054(40)$ & $1.54(43)$ \\
\hline $\mathrm{C} 5$ & $64 \times 16^{3}$ & 1.15 & 1500 & 1.5 & $0.665992(61)$ & $2.32(46)$ & $0.2116(16)$ & $2.38(48)$ \\
\hline D4 & $64 \times 24^{3}$ & 1.15 & 2387 & 1.5 & $0.665927(26)$ & $3.92(79)$ & $0.21478(70)$ & $1.70(24)$ \\
\hline $\mathrm{F} 1$ & $64 \times 32^{3}$ & 1.15 & 2541 & 1.5 & $0.665946(30)$ & $3.37(62)$ & $0.2115(12)$ & $1.06(12)$ \\
\hline G1 & $80 \times 48^{3}$ & 1.15 & 2200 & 1.5 & $0.665943(17)$ & $5.1(1.2)$ & $0.2237(17)$ & $0.637(58)$ \\
\hline $\mathrm{G} 2$ & $80 \times 48^{3}$ & 1.15 & 3436 & 1.5 & $0.665933(18)$ & $5.6(1.8)$ & $0.2235(14)$ & $0.639(26)$ \\
\hline B2 & $24 \times 12^{3}$ & 1.05 & 7819 & 1 & $0.647633(70)$ & $6.79(99)$ & $1.4936(51)$ & $5.80(78)$ \\
\hline C6 & $64 \times 16^{3}$ & 1.05 & 2648 & 1.5 & $0.647645(48)$ & $4.63(96)$ & $1.4389(36)$ & $1.26(14)$ \\
\hline D5 & $64 \times 24^{3}$ & 1.05 & 4000 & 1.5 & $0.647695(37)$ & $3.56(53)$ & $1.3906(45)$ & $0.722(54)$ \\
\hline $\mathrm{F} 2$ & $48 \times 32^{3}$ & 1.05 & 3590 & 1.5 & $0.647680(30)$ & $4.28(74)$ & $1.3708(49)$ & $0.632(45)$ \\
\hline TWA1 & $64 \times 8^{3}$ & 1.15 & 565 & 1.5 & $0.66665(22)$ & $2.8(1.0)$ & $0.5557(96)$ & $0.85(18)$ \\
\hline TWB1 & $64 \times 12^{3}$ & 1.15 & 741 & 1.5 & $0.66590(11)$ & $2.96(96)$ & $0.2709(48)$ & $1.93(50)$ \\
\hline TWC1 & $64 \times 16^{3}$ & 1.15 & 1162 & 1.5 & $0.665990(61)$ & $2.91(73)$ & $0.2484(17)$ & $6.2(2.2)$ \\
\hline TWD1 & $64 \times 24^{3}$ & 1.15 & 2701 & 1.5 & $0.665912(35)$ & $4.63(95)$ & $0.21840(88)$ & $2.43(37)$ \\
\hline OG1 & $80 \times 48^{3}$ & 1.15 & 1248 & 1.5 & $0.643620(16)$ & $1.12(2)$ & $0.22607(58)$ & $6.12(2)$ \\
\hline
\end{tabular}

size of the lattice has been increased to 64 in order to exploit the long plateau in the mesonic correlators. The lattices C5, D4, F1, and G1 are in the regime in which the Polyakov loop has a single peak at zero and is symmetric around it, and the statistics accumulated (of order 2000 configurations) is suitable for the study of the glueball spectrum. The large number of trajectories generated on the B2 lattice is needed because of the larger autocorrelation time observed for this particular choice of parameters. Note that the trajectory length of the HMC for this lattice is $t_{\text {traj }}=1$, shorter than the one for successive runs. The runs on the twisted lattices are designed to check the finitevolume effects in the mesonic spectrum. The statistics is chosen accordingly to yield a good signal in those channels.

\section{A. Observables}

All observables discussed in this paper are obtained from the expectation values of field correlators, using techniques that are standard in lattice simulations. Mesonic observables are extracted from two-point functions of fermion bilinears:

$$
f_{\Gamma \Gamma^{\prime}}(t)=\sum_{\vec{x}}\left\langle\Phi_{\Gamma}(\vec{x}, t)^{\dagger} \Phi_{\Gamma^{\prime}}(\overrightarrow{0}, 0)\right\rangle,
$$

where

$$
\Phi_{\Gamma}(\vec{x}, t)=\bar{\psi}_{1}(\vec{x}, t) \Gamma \psi_{2}(\vec{x}, t)
$$

Note that we always consider nonsinglet flavor states, as indicated by the indices 1,2 that appear in the definition of the fermion bilinear. The matrices $\Gamma$ and $\Gamma^{\prime}$ act in spin space, and determine the quantum numbers of the states that contribute to the correlator in Eq. (1). The channels studied here are obtained by choosing $\Gamma=\Gamma^{\prime}=1, \gamma_{0} \gamma_{5} \gamma_{k}$, $\gamma_{5} \gamma_{k}, \gamma_{0} \gamma_{k}, \gamma_{k}, \gamma_{5}$. The details of the analysis used to extract the Partially Conserved Axial Current (PCAC) mass, the hadron masses, and the decay constants are provided in the appendix of Ref. [55]. Gluonic observables are obtained using the techniques described in Ref. [59].

\section{B. Contamination from excited states}

We had noticed in our previous simulations that lattices with a temporal extent $T / a=16,24$ are not long enough to identify unambiguously the onset of the asymptotic behavior of the field correlators [55]. As discussed below, this is not unexpected if the theory has an IR fixed point. Larger time extensions have been used in this study in order to be able to identify long plateaux in the effective mass plots, and to fit the large time behavior in a regime where contaminations from higher states in the spectrum have died out. A typical set of plateaux for the mass of the pseudoscalar meson is reported in Fig. 1. Lattices with temporal extent $T / a \geq 64$ exhibit long plateaux for the effective mass, which can be fitted to a constant over a large range in $t$. As a consequence, we decided not to use smeared sources for this analysis, since the introduction of smeared sources increases the autocorrelation time of the spectral observables.

\section{Twisted boundary conditions}

In order to investigate in more detail the systematic errors introduced by the finite extent of the spatial volume, we 


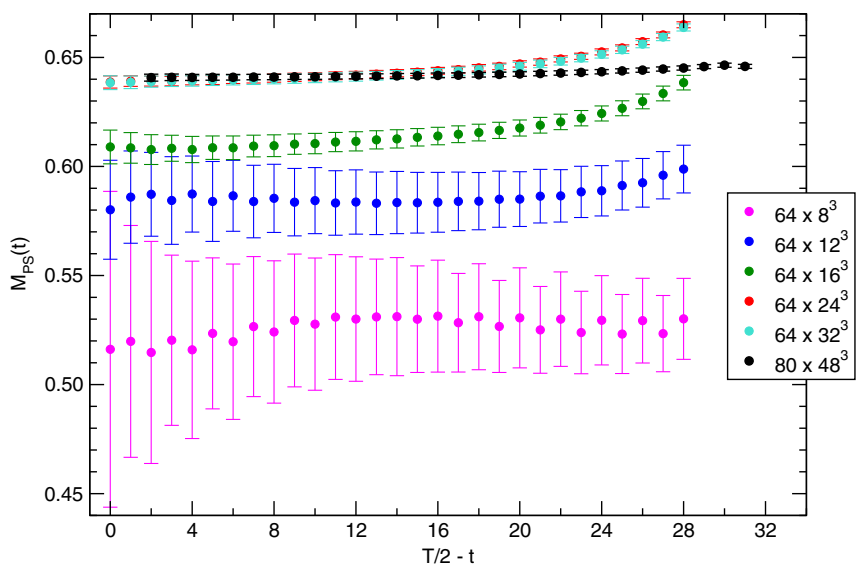

FIG. 1. Plateaux for the effective mass in the PS channel at $\beta=2.25$ and $-a m_{0}=1.15$ for periodic boundary conditions. The figure shows that mass plateaux as a function of $t$ are clearly identified for the current range of parameters, and that there is no contamination from higher states in the spectrum for the lattices considered here, i.e. with $L / a \geq 64$.

have performed a series of simulations with twisted boundary conditions. Twisted boundary conditions, as introduced by 't Hooft [57], can be implemented in a theory with matter in the adjoint representation. We consider here twisted boundary conditions in the spatial directions, which introduce a magnetic flux in the system. In the confined phase, the effect of these boundary conditions should vanish as the spatial size of the box is taken to infinity. Any difference in the spectrum as the boundary conditions are varied could signal sizable finitevolume effects.

Let us summarize the implementation of the boundary conditions used in this work. Twisted boundary conditions are imposed for both the gauge and the fermion fields:

$$
\begin{gathered}
A_{\mu}\left(x_{0}, \boldsymbol{x}+L \boldsymbol{e}_{k}\right)=\Omega_{k} A_{\mu}\left(x_{0}, \boldsymbol{x}\right) \Omega_{k}^{\dagger}, \\
\psi\left(x_{0}, \boldsymbol{x}+L \boldsymbol{e}_{k}\right)=\Omega_{k} \psi\left(x_{0}, \boldsymbol{x}\right) \Omega_{k}^{\dagger},
\end{gathered}
$$

where $\Omega_{k}$ are generic elements of the gauge group. For the fields to be single valued, the following constraint must be satisfied:

$$
\Omega_{j} \Omega_{k}=e^{\frac{2 \pi i}{N} c_{j k l} m_{l}} \Omega_{k} \Omega_{j},
$$

where $m_{l} \in \mathbb{Z}$ if the gauge group is $\mathrm{SU}(\mathrm{N})$. Twisted boundary conditions introduce three orthogonal Dirac strings aligned along the $\boldsymbol{e}_{1}, \boldsymbol{e}_{2}$ and $\boldsymbol{e}_{3}$ directions, each one carrying center-magnetic flux equal to $m_{1}, m_{2}$ and $m_{3}$ respectively. Note that physical observables only depend on the magnetic flux $\boldsymbol{m}$, and not on the particular choice of the $\Omega_{k}$ matrices.

For the simulations presented here, i.e. for the case $N=2$, we choose the maximally symmetric twist:

$$
\boldsymbol{m}=(1,1,1),
$$

which preserves the octahedral isometries (including parity) and charge-conjugation symmetry.

Twisted boundary conditions are implemented on the lattice by modifying the action. The link variables and fermions are still periodic, but the gauge part of the action is modified by multiplying the plaquettes by a center element. For a theory with $\mathrm{SU}(2)$ gauge group

$$
S_{g}=-\frac{\beta}{4} \sum_{x, \mu<\nu} z_{\mu \nu}(x) \operatorname{tr} U_{\mu \nu}(x) .
$$

The contribution to the action of a given plaquette is multiplied by -1 , if the plaquette is pierced by the Dirac string, i.e., with $\mu, \nu$ and $\rho$ the three spatial directions and indicating with zero the temporal direction:

$$
z_{\mu \nu}(x)=\left\{\begin{array}{ll}
-1 & \text { for } x_{0}=x_{\rho}=1 \\
1 & \text { otherwise }
\end{array},\right.
$$

for the three possible choices of $\mu<\nu$. The fermion part of the action remains unchanged, and therefore only the gluonic force is modified in the molecular dynamics evolution.

\section{Open boundary conditions}

In a setup with periodic boundary conditions in all directions, the autocorrelation times increase exponentially with the lattice spacing because of the existence of topological sectors in the continuum limit. Neumann (open) boundary conditions remove the infinite-energy barrier between topological sectors. For the range of lattice spacings studied in Ref. [60] in the case of QCD, the autocorrelation times scale with $a^{-2}$.

Even though our simulations are performed at fixed $\beta$, we have observed a very poor scaling of the autocorrelation time of the topological susceptibility towards the chiral limit (which might be a peculiarity of IR conformality). Simulations with open boundary conditions have been performed using a modified version of the HiRep code, showing that indeed the choice of boundary conditions ameliorates this problem.

We have generated only one lattice with open boundary conditions, denoted OG1 in Table I. The parameters for this run correspond to the ones used for the runs G1 and G2 with periodic boundary conditions. These are our largest lattices at the lighter fermion mass. This extra set of simulations has been added to address specifically the problems that we observe in the glueball spectrum at the lighter mass. As the glueballs are the lightest states in this theory, it is important to have a quantitative understanding of the finite-volume effects for these observables.

As in the case of periodic boundary conditions, spectral quantities can be extracted from two-point correlators of 
composite fields with the appropriate quantum numbers. This follows from the observation that open boundary conditions correspond in Hamiltonian formalism to the insertion of a pure state with quantum numbers of the vacuum; see e.g. Refs. $[58,61,62]$ for a recent discussion. We notice that in this case the two-point functions cannot be averaged over time, as translational invariance is lost in the time direction. The details of our analysis are presented in the following section.

\section{RESULTS}

\section{A. Heavier fermion mass}

The heavier fermion mass in the new set of simulations is $a m_{0}=-1.05$, which corresponds to a PCAC mass of $a m=0.2688(15)$. The full spectrum is shown in Fig. 2. As observed in previous studies the mesons are heavier than the glueballs, with the smallest scale being set by the string tension. On the largest volumes, we observe that the lightest glueball state is in the $A^{++}$channel (which corresponds to the continuum $0^{++}$) and the ground states in the $E^{++}$and in the $T^{++}$channels (which both single out the continuum $2^{++}$) have the expected degeneracy. It is worth noting that the string tension can be obtained from correlators of Polyakov loops wrapping either in the temporal or in the spatial direction; the two measures are characterized by finite-volume effects driven by the spatial or the temporal size respectively. In Fig. 2 and later in Fig. 4 we report both measures as a function of their relevant size.

The detailed analysis of the mesonic spectrum is shown in Fig. 3. At this value of the fermion mass, the finitevolume effects on the mesonic states are small, and we can confidently extrapolate to the thermodynamical limit. Different combinations of $\Gamma, \Gamma^{\prime}$ that project onto the same physical states yield results that are compatible within

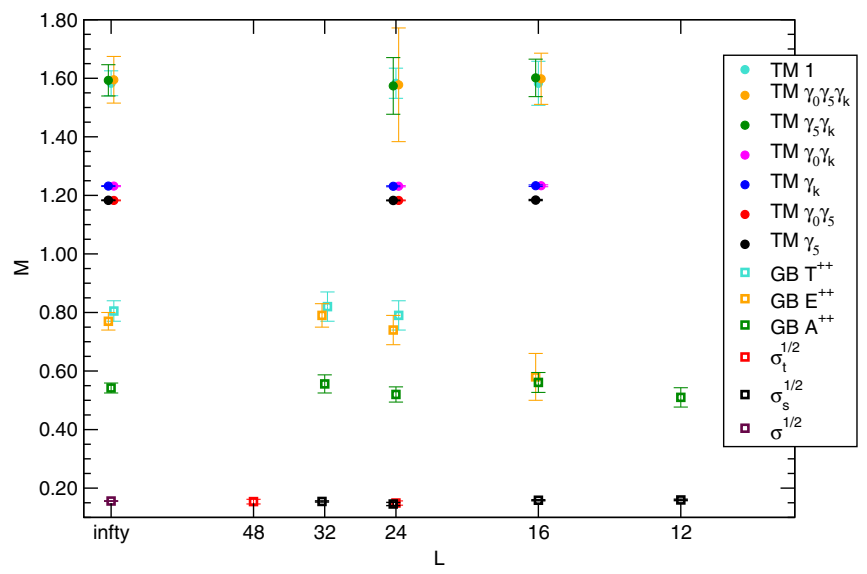

FIG. 2. Summary of the spectrum for all the states included in this analysis at $a m_{0}=-1.05$ with periodic boundary conditions. The data are shown as a function of the number of sites in the spatial directions $L / a$.

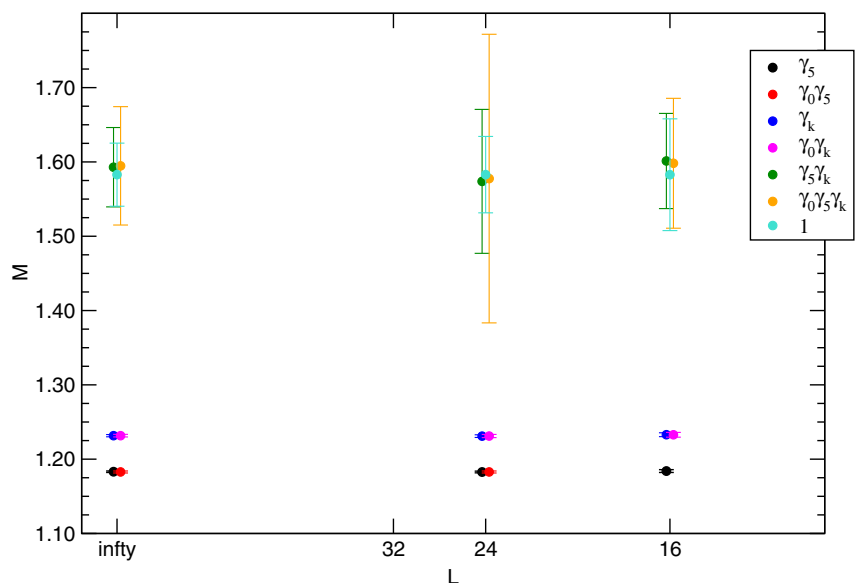

FIG. 3. Summary of the spectrum of the mesonic states included in this analysis at $a m_{0}=-1.05$ with periodic boundary conditions. The data are shown as a function of the number of sites in the spatial directions $L / a$.

statistical errors. The new simulations confirm the results that we presented in previous studies [55].

The results for the string tension are reported in Fig. 4. Simulations on sufficiently large lattices, $L / a \geq 24$, show a satisfactory agreement between the spatial and temporal string tensions.

\section{B. Lighter fermion mass}

Let us now consider the lightest mass, where the finitevolume effects are expected to be larger. The mass of the pseudoscalar, reported in Fig. 1, is affected by sizable finite-volume corrections on the smaller lattices; these corrections can be as large as $23 \%$ if $L M_{\mathrm{PS}} \simeq 4$ and a very visible $5 \%$ for $L M_{\mathrm{PS}} \simeq 9.75$. The fitted value seems to converge for lattices with $L / a \geq 24$, corresponding to

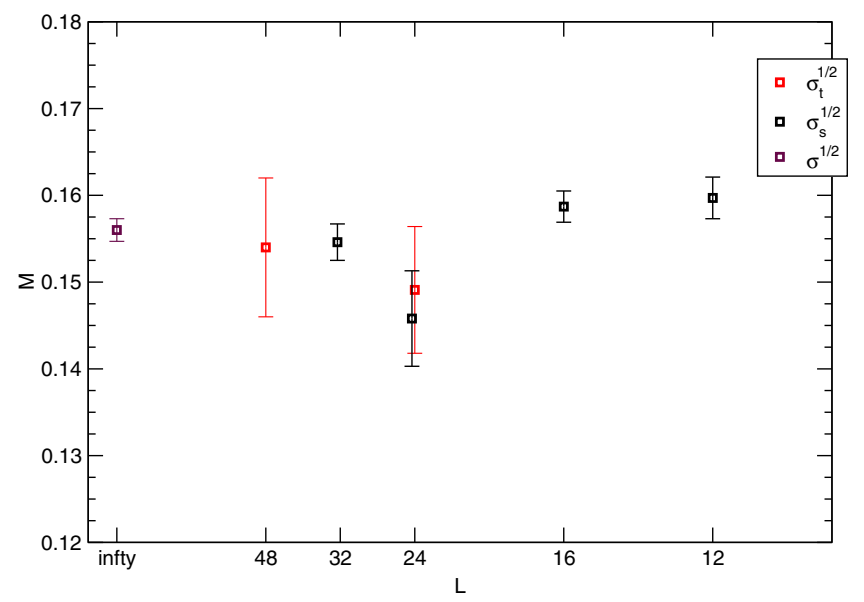

FIG. 4. String tension extracted from spatial and temporal Polyakov loops at $a m_{0}=-1.05$ with periodic boundary conditions. The results are shown as a function of the number of sites in the spatial directions $L / a$. 
$L M_{\mathrm{PS}} \simeq 15.4$. Moreover, this plot shows that the effective mass is growing as the spatial size of the lattice is increased. This is consistent with the observation that, in a theory where chiral symmetry is not spontaneously broken, the pseudoscalar mass reaches the infinite-volume limit from below, as explained in Refs. [63-65].

The plateaux in the effective mass of the pseudoscalar are reported as a function of the time separation between the interpolating operators $t$. They show that a large temporal extent is also required in order to suppress the corrections from heavier states in the same channel. This is expected for a mass deformed conformal theory, since all the states in the spectrum should scale to zero following the same power-law scaling formula. The contribution from excited states becomes negligible when

$$
t \Delta M_{\mathrm{PS}} \gg 1
$$

where $\Delta M_{\mathrm{PS}}$ is the mass difference between the ground state and the first excited stated in the pseudoscalar channel. For a conformal theory near the chiral limit, $\Delta M_{\mathrm{PS}} \propto M_{\mathrm{PS}}$, so that the exponential suppression of the excited states becomes really effective only at large temporal separations.

The data for the full spectrum including the glueballs are shown in Fig. 5. Note that in this case the glueball spectrum shows a lack of degeneracy between the ground-state masses in the $E^{++}$and in the $T^{++}$channels and a neardegeneracy of the $A^{++}$channel and of the $E^{++}$, with the latter mass having the tendency to be lighter. This is at odds with the expected behavior as the continuum limit is approached, where the $E^{++}$and $T^{++}$channels should become degenerate, and heavier than the scalar state $A^{++}$. This unexpected behavior has already been observed in Ref. [31], where it was ascribed to finite-size effects arising at the crossover between the small volume regime and the

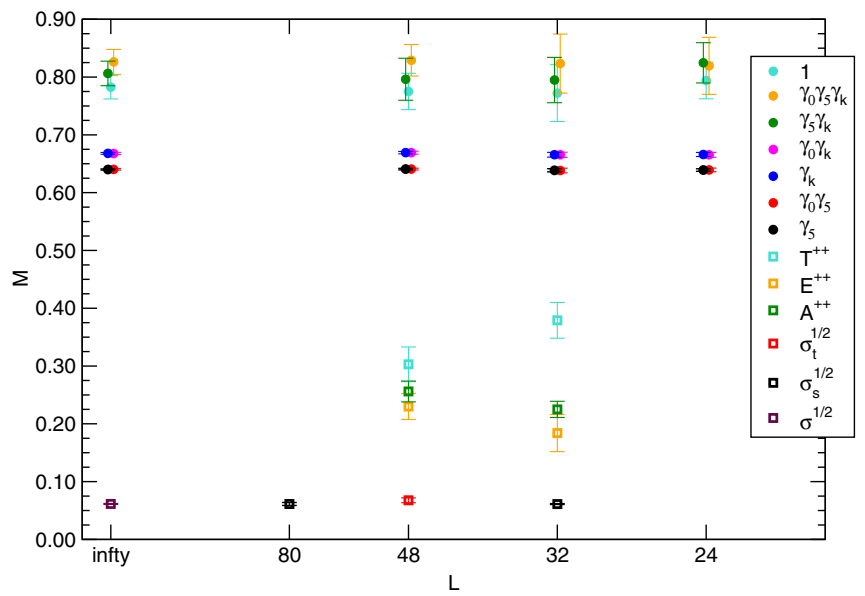

FIG. 5. Summary of the spectrum for the lattices included in this analysis at $a m_{0}=-1.15$ with periodic boundary conditions. The data are shown as a function of the number of sites in the spatial directions $L / a$. regime connected to the thermodynamic limit. This interpretation is supported by our current study at higher mass, where hints of those phenomena can be seen on the smallest lattices, but then disappear when the size of the lattices is increased, as shown in Fig. 2.

As discussed above, finite-volume effects can be investigated by comparing results obtained with different boundary conditions; in particular for the glueball spectrum the comparison between periodic and open boundary conditions yields interesting results. The spectrum is obtained from the large time behavior of correlators far away from the boundaries, while trying to average over different times in order to improve the statistical signal. More precisely, we computed the correlators,

$$
\begin{aligned}
f_{12}(t)= & \frac{1}{N_{\mathrm{pts}}} \sum_{n=-\left\lfloor N_{\mathrm{pts}} / 2\right\rfloor+1}^{\left\lfloor N_{\mathrm{pts}} / 2\right\rfloor}\left\langle\mathcal{O}_{1}(T / 2+n+t / 2) \mathcal{O}_{2}(T / 2\right. \\
& +n-t / 2)\rangle,
\end{aligned}
$$

with a straightforward modification in the case where $t$ is odd. For a given time separation $t$ between the interpolating fields $\mathcal{O}_{1}$ and $\mathcal{O}_{2}$, we average over $N_{\text {pts }}$ time planes; the values of $N_{\text {pts }}$ are chosen so that the $T / 2+\left\lfloor N_{\text {pts }} / 2\right\rfloor+t / 2$ and $T / 2-\left\lfloor N_{\mathrm{pts}} / 2\right\rfloor+1-t / 2$ are at a distance of at least seven lattice spacings from the boundary.

The results for the correlator in the $E^{++}$channel are shown in Fig. 6. The averaging over several time planes improves the determination of the correlators, and the effective mass plot shows a plateau for time separations $t \geq 4$. Although we analyzed several values of $N_{\mathrm{pts}}$, we
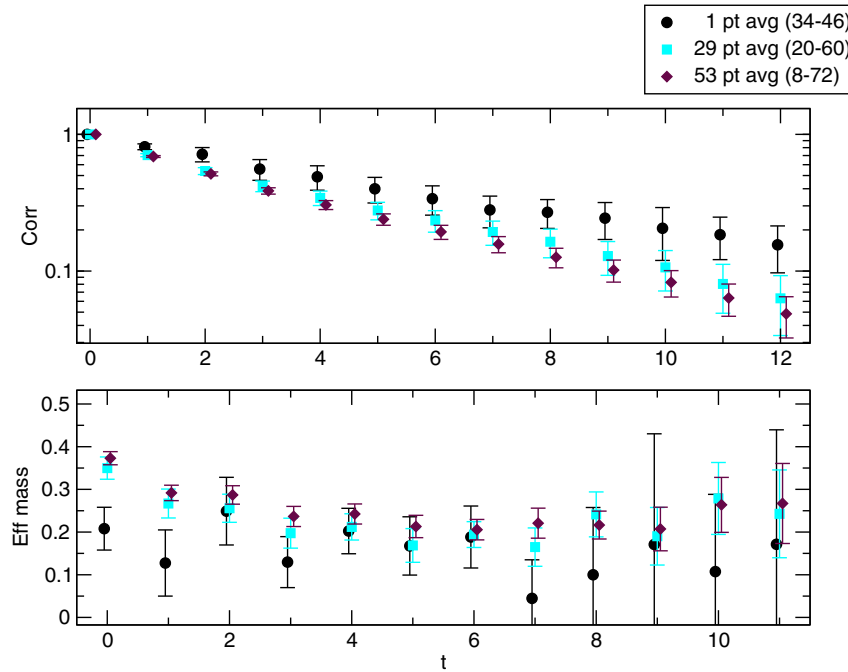

FIG. 6. The correlator $f_{12}(t)$ defined in Eq. (10) (top) and the corresponding effective mass (bottom) for the $E^{++}$glueball as a function of the time separation $t$. Simulations are performed with open boundary conditions. Different symbols correspond to averages over different numbers of time planes, i.e. to choosing $53,29, N_{\mathrm{pts}}=1$. 
TABLE II. Results for the glueball spectrum at $a m=-1.15$. We show the results obtained with periodic boundary conditions (PBC), and with open boundary conditions (OBC) averaged over $N_{\text {pts }}=29$ time slices, as explained in the text. As discussed in Sec. III B the determination of the statistical error with PBC is most likely underestimated because of long autocorrelation times.

\begin{tabular}{lrrr}
\hline \hline & $A^{++}$ & $E^{++}$ & $T^{++}$ \\
\hline OBC & $0.179(28)$ & $0.212(34)$ & $0.283(27)$ \\
PBC & $0.256(18)$ & $0.230(23)$ & $0.303(30)$ \\
\hline \hline
\end{tabular}

discuss in detail the results for $N_{\mathrm{pts}}=29$. Other channels show a similar behavior for both the correlators and the effective masses.

The results for the glueball spectrum are summarized in Table II, where they are compared to the ones obtained with periodic boundary conditions. The table shows a discrepancy for the mass of the $A^{++}$glueball. We will argue that the error for the case with periodic boundary conditions may be underestimated because of very long autocorrelation times. The expected ordering of the masses, with the scalar glueball lighter than its spin-2 counterpart, is observed when using open boundary conditions. The picture is much less clear in the case of periodic boundary conditions: the $A^{++}$state is heavier than the $E^{++}$, although the values are compatible within errors. For both choices of the boundary conditions, we do not observe the expected degeneracy between the $E^{++}$and $T^{++}$channels. Note that this degeneracy is only expected in the continuum limit; our simulations being performed at only one value of the lattice spacing, we cannot disentangle finite-volume effects from the $O(a)$ lattice artifacts. However, the fact that the $E^{++}$ and the $T^{++}$glueballs are degenerate at the heavier value of the mass suggests that the discrepancy for lighter fermions is due to finite-volume effects. In order to understand better the origin of these different patterns, we have investigated the integrated autocorrelation time of the topological charge in both settings, since a poor sampling of the
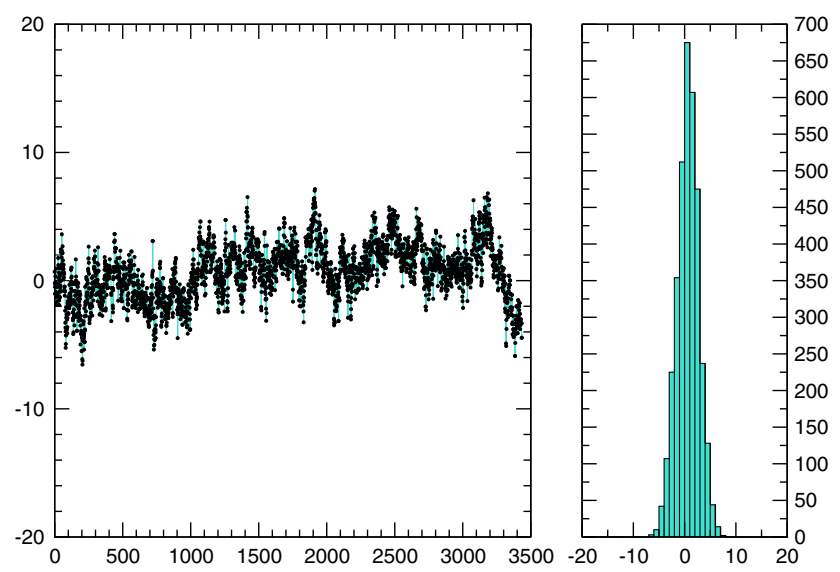

topological sectors could in principle lead to larger systematic errors. We measured the topological charge using a bosonic estimator computed along the Wilson flow:

$$
Q_{t}=-\frac{a^{4}}{32 \pi^{2}} \sum_{x} \epsilon_{\mu \nu \rho \sigma} \operatorname{tr}\left[G_{t, \mu \nu}(x) G_{t, \rho \sigma}(x)\right],
$$

where $G_{t, \mu \nu}(x)$ is the clover term for the field strength tensor on the lattice constructed from the gauge links at flow time $t$ [60]. The time histories for the topological charge defined from the gauge fields at flow time $t=2.0$ in the runs with periodic and open boundary conditions, G2 and OG1 respectively, are shown in Figs. 7. The two plots suggest that the topological sampling is much more efficient in the simulations with open boundary conditions. A more quantitative assessment of the tunneling between topological sectors can be obtained by computing the integrated autocorrelation time for both runs. We use the Madras-Sokal definition of the autocorrelation time [66], implemented according to Refs. [67,68]. As an illustration of its typical behavior, we report in Fig. 8 the normalized autocorrelation function for the topological charge computed at flow time $t=2.0$, as a function of the time lag $\xi$ between measurements:

$$
\bar{\Gamma}(\xi)=\frac{1}{N-\xi} \sum_{i=1}^{N-\xi}\left(Q_{i}-\bar{Q}\right)\left(Q_{i+\xi}-\bar{Q}\right),
$$

where $N$ is total number of measurements, $Q_{i}$ denotes the $i$ th measurement of the topological charge $Q$, and $\bar{Q}$ its average. The data show that the correlation decays much faster for the case of open boundary conditions. The integrated autocorrelation time $\tau_{\text {int }}$ is defined as

$$
\tau_{\text {int }}=\frac{1}{2}+\sum_{\xi=1}^{W} \bar{\Gamma}(\xi) / \bar{\Gamma}(0),
$$
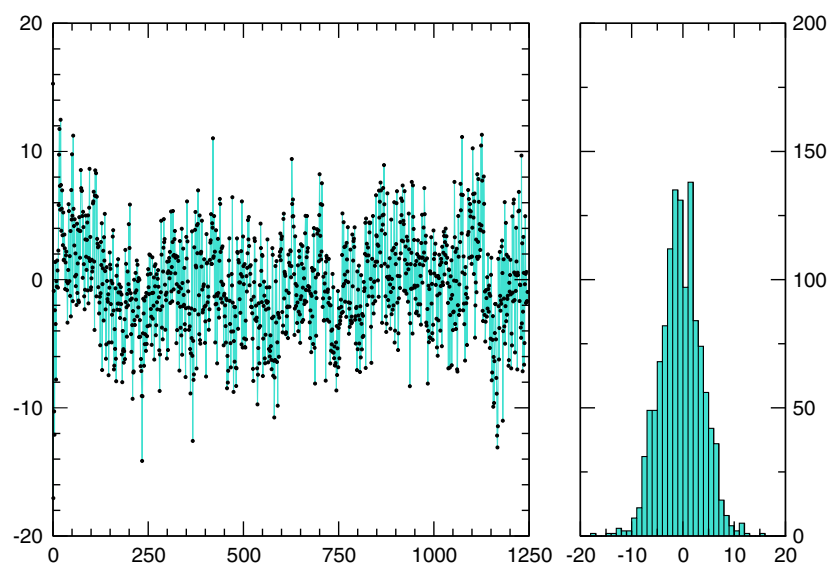

FIG. 7. Time history for the topological charge defined from the bosonic estimator in Eq. (11) for the run G2, with periodic boundary conditions (left), and for the run OG1, with open boundary conditions (right). The topological charge is measured at flow time $t=2.0$. 
DEL DEBBIO, LUCINI, PATELLA, PICA, and RAGO

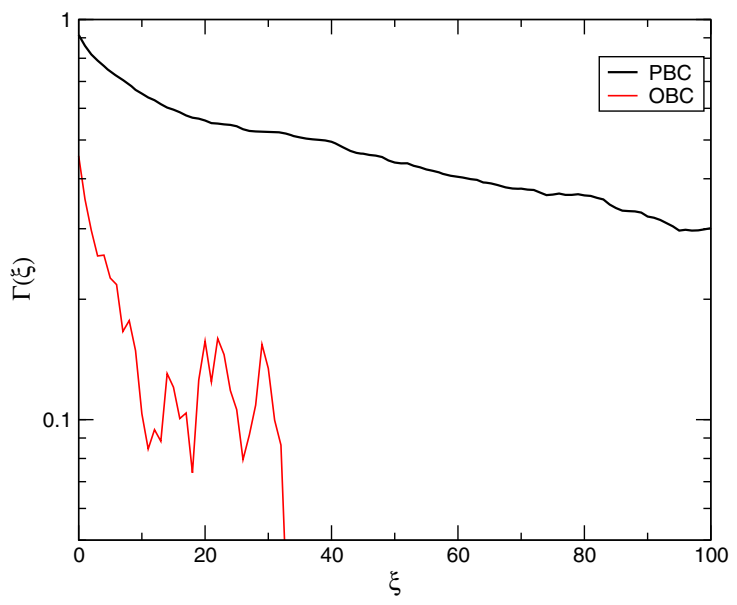

FIG. 8. Normalized correlation function $\bar{\Gamma}(\xi)$ for the topological charge as a function of the time lag between measurements $\xi$. The black (respectively red) curve corresponds to the data obtained with periodic (respectively open) boundary conditions.

where $W$ is the size of the Madras-Sokal window. The integrated autocorrelation times, which are reported in Table III, reflect the longer correlations in the case of the periodic boundary conditions. The current runs on the periodic lattices are not long enough to determine with confidence the autocorrelation time. However, it is clear from the numbers obtained for $\tau_{\text {int }}$ using the Madras-Sokal procedure, and from the plot of the normalized correlation, that the autocorrelation is much longer in the case of the periodic boundary conditions than it is with open boundary conditions.

In the light of these results, it is tempting to associate the unexplained features of the glueball spectrum to the lack of decorrelation between topological sectors in the case of simulations with periodic boundary conditions. A more detailed investigation of the correlation between topology and the glueball spectrum is beyond the scope of this paper, and is postponed to future investigations. Here we will simply conclude that the simulations with periodic boundary conditions are affected by systematic errors in the glueball spectrum that we cannot fully quantify currently. It is reassuring to note that the limited data we have with open boundary conditions yield results that are in line with the expected ones in the continuum limit.

TABLE III. Autocorrelation time of the topological charge; for each choice of boundary conditions, the autocorrelation is computed for two values of the flow time $t$.

\begin{tabular}{lcc}
\hline \hline$t$ & b.c. & $\tau_{\text {int }}$ \\
\hline 2.0 & OBC & $6.0(2.0)$ \\
2.0 & PBC & $80.6(51.2)$ \\
5.0 & OBC & $7.8(2.7)$ \\
4.4 & PBC & $87.4(54.7)$ \\
\hline \hline
\end{tabular}

PHYSICAL REVIEW D 93, 054505 (2016)

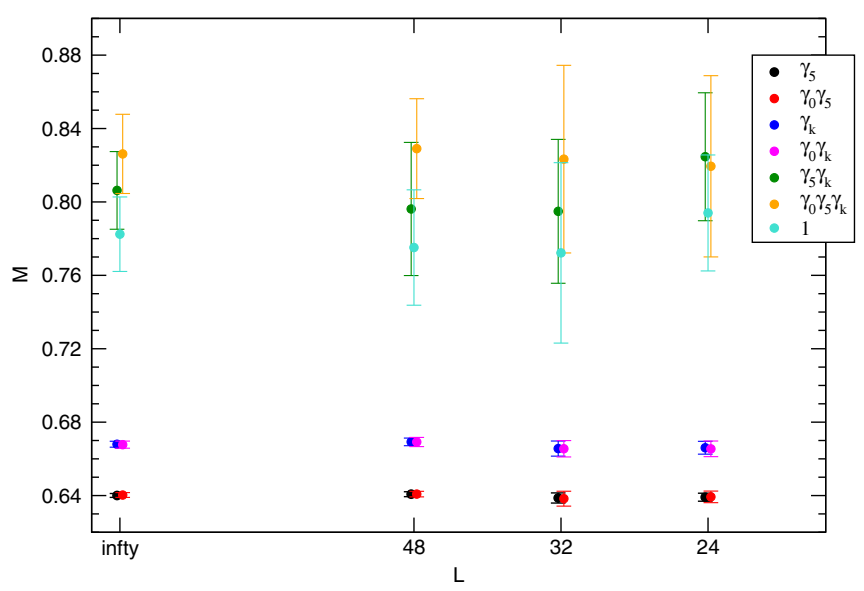

FIG. 9. Summary of the spectrum of the mesonic states for the lattices included in this analysis at $a m_{0}=-1.15$ for periodic boundary conditions. The data are shown as a function of the number of sites in the spatial directions $L / a$.

As already noted in previous studies, the meson spectrum is found to reach its asymptotic value for smaller volumes than the glueballs. We can therefore focus on a more quantitative analysis of the mesonic spectrum at $a m_{0}=-1.15$, which, for clarity, is reported on a larger scale in Fig. 9. The data show that the finite-volume effects for this value of the mass are nicely under control, and that a reliable infinite-volume limit can be taken. In order to get a further estimate of the size of finite-volume effects, we compare the mass obtained on increasingly large volumes using periodic and twisted boundary conditions. The results of these comparisons are reported in Fig. 10, where independence from the boundary conditions is seen for $L / a \geq 24$. We conclude from this analysis that systematic errors due to finite-volume effects become smaller than $1 \%$ for $L M_{\mathrm{PS}} \geq 15$.

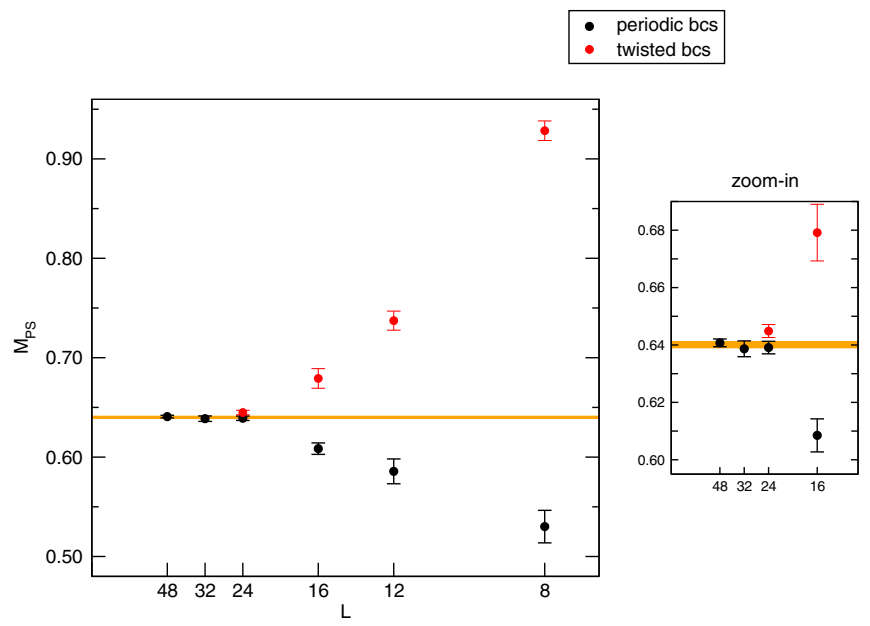

FIG. 10. Comparison of the pseudoscalar mass obtained from simulations with periodic and twisted boundary conditions respectively at $a m_{0}=-1.15$. 
- periodic bcs

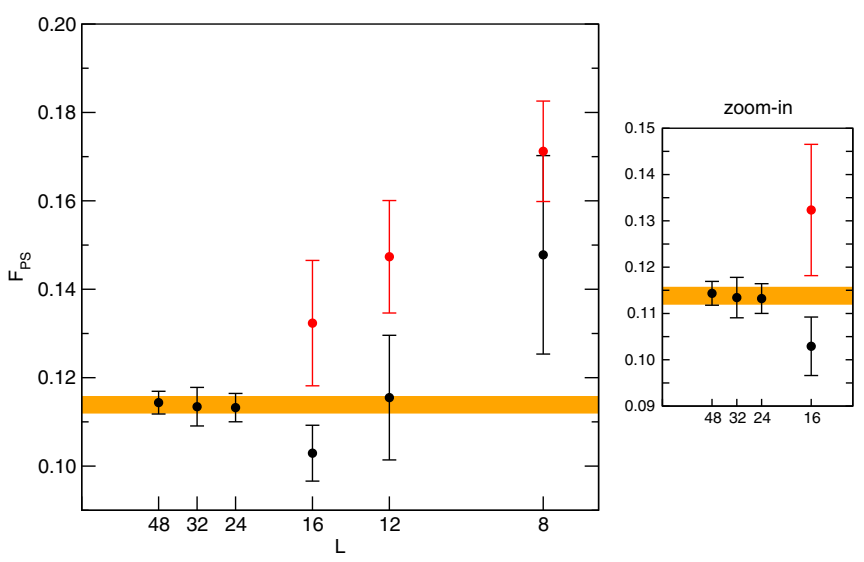

FIG. 11. Pseudoscalar decay constant as a function of the lattice size $L / a$ at $a m_{0}=-1.15$.

Our previous studies had also highlighted a strong dependence of the pseudoscalar decay constant on the size of the lattice. In order to get a more quantitative understanding of the problem, we have measured $f_{\text {PS }}$ on the larger lattices used for this analysis. The results are shown in Fig. 11. The situation for the decay constant is more subtle: the behavior is not monotonic as a function of the lattice size, and we do not have a theoretical description of the observed pattern. This unusual behavior is due to the value obtained at $L / a=16$, which is only $2 \sigma$ away from the value obtained from the larger lattices. An extrapolation based on the data for $L / a<24$ would be affected by large systematic errors, when compared to the value we obtain if only the larger volumes are used. In particular the extrapolated value obtained from the smaller lattices would end up being much smaller than the one extracted from the large lattices, hence biasing the characterization of the large-distance behavior of the theory under study. It is worthwhile to emphasize once again the importance of simulating near-conformal theories in sufficiently large volumes. For each individual theory, and for each choice of bare parameters in the action, the minimal volume needs to be determined by careful numerical analyses.

The data for the rest of the spectrum follow the pattern observed in the examples above. For the mass range explored in this study, the finite-volume effects are below the $1 \%$ level provided the spatial size of the lattice is such that $L M_{\mathrm{X}} \geq 5$. The mass $M_{\mathrm{X}}$ that determines the minimum size of the lattice is the lightest mass in the spectrum. Note that for our lattices the lightest state is always a glueball state. As a rule of thumb, we can translate the bound above into a constraint for the mass of the pion, which is approximately three times heavier than the glueball; the condition to avoid finite-volume effects becomes $L M_{\mathrm{PS}} \geq 15$.

In the regime where finite-volume effects are below $1 \%$, we can study the ratio of masses of different states in the

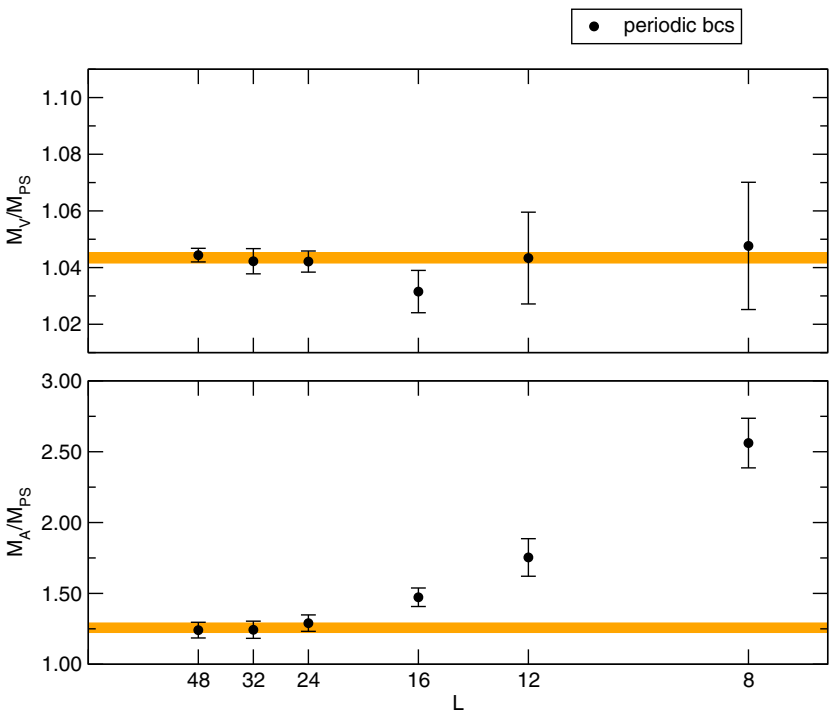

FIG. 12. Ratios of the mass of the vector state (upper plot) and axial vector state (lower plot) to the mass of the pseudoscalar state. The data are obtained at the lightest mass, and plotted as a function of the lattice volume $L$.

spectrum. A consequence of the existence of an IRFP is that all states in the spectrum have to scale to zero as a function of the fermion mass, at a common rate dictated by the mass anomalous dimension $\gamma_{*}$ :

$$
M_{X} \propto m^{1 /\left(1+\gamma_{*}\right)}
$$

where $X$ denotes a generic state in the spectrum of the theory. This simple scaling law implies that the spectrum of a theory with an IRFP is different from the spectrum of QCD already at a qualitative level. The lack of spontaneous symmetry breaking in a conformal theory yields the absence of light Goldstone bosons, and hence the ratio of the Goldstone boson mass to the mass of other states in the spectrum does not vanish.

The data presented in Ref. [31] suggest that the mass of the pseudoscalar state scales indeed at the same rate as the vector mass for $\mathrm{SU}(2)$ with adjoint fermions, thereby going to a constant in the zero-mass limit. However some dependence on the finite size of the lattice remains visible for the smallest lattices. The recent runs on larger volumes presented in this paper have settled this issue for the two masses that we have considered. Figure 12 shows the ratio of the mass of the vector and axial vector states to the mass of the pseudoscalar state for the lightest mass simulated. The results for the ratio become independent of the lattice size in both channels for $L>24$.

\section{Extrapolated results}

The data from the larger lattices can be extrapolated to the thermodynamical limit. We used a fit to a constant including the data from lattices with linear size $L \geq 24$ for 
TABLE IV. Summary of the infinite-volume spectrum of the theory for both values of the fermion mass. The values reported in the table are obtained by extrapolating the results from our largest lattices. Given the systematic errors that are still visible in the glueball spectrum, we do not provide extrapolated values for the mass of the $E^{++}$and the $T^{++}$states for the lighter fermion mass. All values are given in units of the lattice spacing.

\begin{tabular}{|c|c|c|c|c|c|c|c|c|c|c|c|}
\hline$-a m_{0}$ & $\sqrt{\sigma}$ & $A^{++}$ & $E^{++}$ & $T^{++}$ & $\gamma_{5}$ & $\gamma_{0} \gamma_{5}$ & $\gamma_{0} \gamma_{k}$ & $\gamma_{k}$ & 1 & $\gamma_{5} \gamma_{k}$ & $\gamma_{0} \gamma_{5} \gamma_{k}$ \\
\hline 1.05 & $0.156(1)$ & $0.542(17)$ & $0.77(3)$ & $0.80(4)$ & $1.183(1)$ & $1.183(1)$ & $1.232(2)$ & $1.232(1)$ & $1.583(42)$ & $1.593(53)$ & $1.595(80)$ \\
\hline 1.15 & $0.0614(8)$ & $\ldots$ & $\ldots$ & $\ldots$ & $0.6401(11)$ & $0.06402(13)$ & $0.6677(19)$ & $0.6680(16)$ & $0.7824(20)$ & $0.806(21)$ & $0.826(22)$ \\
\hline
\end{tabular}

the light mass, and $L \geq 16$ for the heavy one. The results of these fits are already reported in the figures presented above. We summarize the results for the extrapolated values for both values of the fermion mass in Table IV. All values are given in units of the lattice spacing. It is interesting to point out that the degeneracies in the spectrum follow the expected pattern, both for the glueball sector and the mesonic sector. This suggests that the lattice artifacts are relatively small in our simulations, and the discrepancies from the expected spectrum vanish as long as we can take the thermodynamical limit. In some channels, like e.g. the axial vector, the discrepancy between the result at $L / a=$ 16 and the extrapolated result at infinite volume is as large as $16 \%$.

The scaling dictated by the existence of the IRFP [see e.g. Eq. (14)] should be obeyed by the extrapolated spectrum. Figure 13 shows that the scaling ratio

$$
(a M) /(a m)^{1 /\left(1+\gamma_{*}\right)}
$$

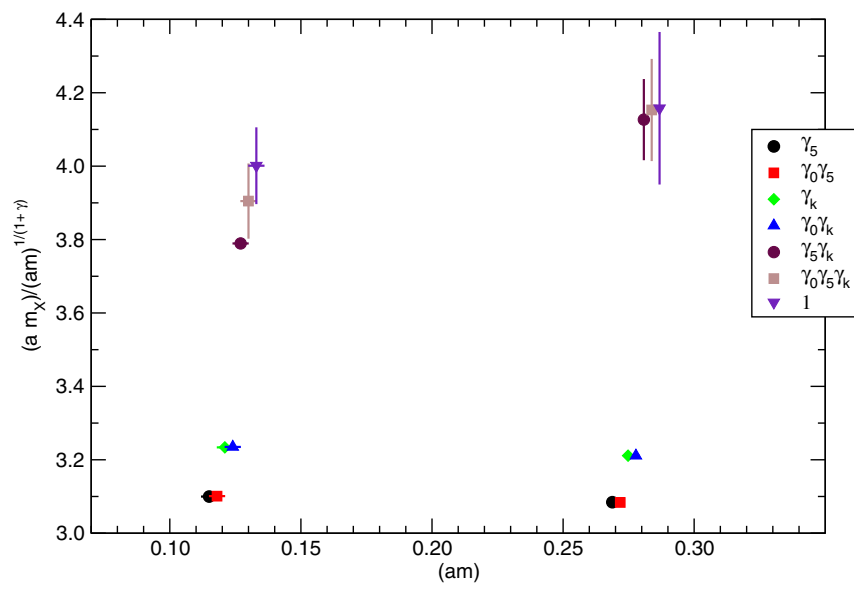

FIG. 13. Scaling behavior of the mesonic spectrum. The mass of the state $X$ is denoted by $M_{X}$, while $m$ denotes the bare mass of the fermionic field. The rescaled data become independent of the value of the fermion mass, which is in agreement with the expected behavior in the presence of an IRFP. Different colors correspond to different quantum numbers of the mesons. Some points are shifted along the horizontal axis for clarity. Note that the scaling exponent is not determined by a fit to the data; its value is taken from existing studies in the literature, as explained in the text. remains constant for both values of the fermion mass, as expected from the scaling hypothesis. Note that the value of the critical exponent $\gamma_{*}=0.371$ is taken from Ref. [38], and is not adjusted to the results of the current simulations.

\section{Finite-size scaling}

In the presence of an IR fixed point, the dependence of the spectrum and matrix elements on the fermion mass and volume is determined by finite-size scaling (FSS). For sufficiently small fermion masses and sufficiently large volumes the dependence of field correlators is determined by scaling relations that are obtained from the linearized RG equations in a neighborhood of the fixed point. The scaling laws for the theory under consideration here were studied in detail in Refs. [31-34,69]. Here we simply recall the results for the masses in the spectrum:

$$
L M_{X}=f_{X}(x),
$$

where $M_{X}$ is the mass of the physical state $X, x=L^{y_{m}} m$ is the so-called scaling variable, and $y_{m}=1+\gamma_{*}$. The function $f_{X}$ is a universal function, in the sense that data generated at different values of the gauge coupling, fermion mass, and volume should all be described by the same function. The only theoretical constraint on $f_{X}$ comes from

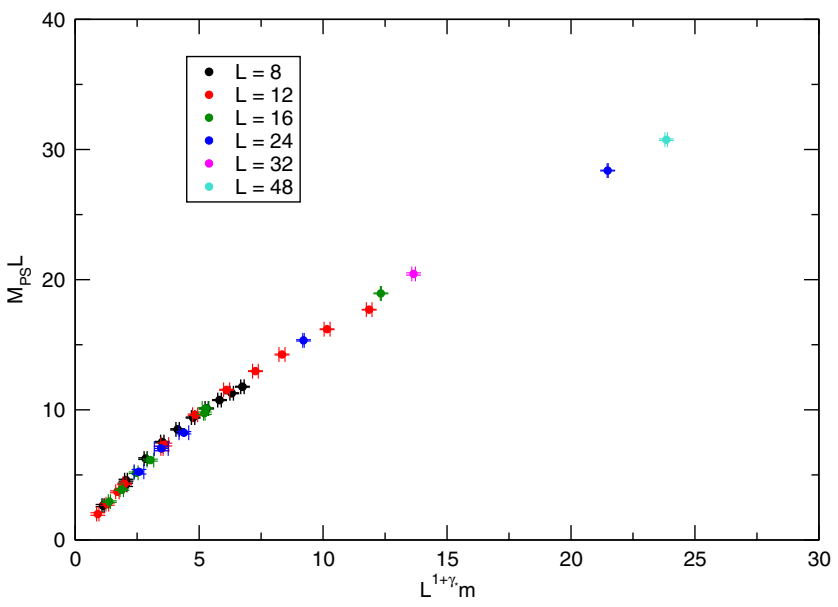

FIG. 14. Finite-size scaling of the pseudoscalar mass. The data obtained on different volumes combined using the scaling exponent determined in Ref. [38] fall on a universal curve as expected. 


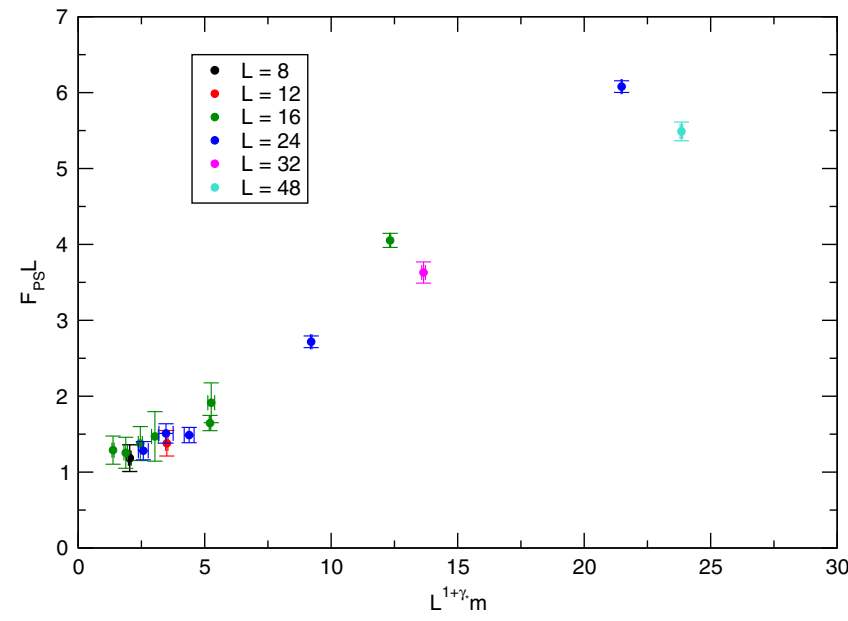

FIG. 15. Finite-size scaling of the pseudoscalar decay constant. The data obtained on different volumes combined using the scaling exponent determined in Ref. [38] fall on a universal curve; however the scaling deviations for the decay constant are larger than the ones observed for the pseudoscalar mass.

the fact that it should reproduce the infinite-volume scaling with the mass of the fermion as $L$ becomes large. It is easy to verify that

$$
f_{X}(x) \stackrel{x \vec{\infty}}{\sim} A_{X} x^{1 / y_{m}}
$$

satisfies this condition.

For each observable we expect a different function, but then that single function must describe the behavior of data from lattices with different volumes. The data shown in Figs. 14 and 15 are in excellent agreement with the scaling dictated by the renormalization group [70], thereby strengthening the hypothesis that this theory does indeed possess a fixed point that governs the large-distance dynamics. The scaling of the variables with volumes is obtained using the critical exponent $\gamma_{*}$ determined in Ref. [38] from the spectrum of the Dirac operator. Once again note that $\gamma_{*}$ is not a free parameter in this study; it is instead fixed to the best value available in the literature.

\section{CONCLUSIONS}

In this paper we have reported the results of our latest large-volume runs for the spectrum of $\mathrm{SU}(2)$ gauge theory with two adjoint fermions. This study was motivated by the finite-volume effects seen in previous simulations, which were potentially obscuring the scaling expected for a theory with an IR fixed point of the RG flow.

The data show that large volumes are mandatory for a robust quantitative determination. We have shown that smaller volumes are enough to control finite-size effects on the triplet meson masses; however much larger volumes are needed in order to have a good control over glueball finite-size effects. This is not surprising considering that this theory shows a separation of scales between the two sectors.
Finite-volume effects on masses of triplet mesons drop below $5 \%$ if $L M_{\mathrm{PS}} \simeq 9.5$. We have substantiated this statement with a systematic comparison of triplet meson masses calculated for different values of the spatial volume, with periodic and twisted boundary conditions. In the gluonic sector our conclusions are less definite, because of the intrinsic difficulty in measuring glueball masses. As a rule of thumb, a spatial size such that $L \sigma^{1 / 2} \gtrsim 3$ is desirable in order to avoid large finite-volume effects in the gluonic sector.

We have analyzed in detail two values of the fermion mass, which produce compatible ratios of triplet-meson masses. These findings strengthen our previous conclusions on the existence of a fixed point for this theory. The fermion masses used in this study seem to be light enough in order for the triplet meson spectrum to be in the scaling region of the IR fixed point. Moreover the data obtained on different volumes fall on a universal curve using the value of the mass anomalous dimension previously determined in Ref. [38]. On the other hand our infinite-volume estimate for the ratio $M_{\mathrm{PS}} / \sqrt{\sigma}$ changes substantially with the fermion mass, suggesting that lighter masses are necessary in order to access the scaling region of the gluonic spectrum.

Should such theories become serious candidates for phenomenology, we have presented convincing evidence that physically relevant information on their spectrum can only be extracted from large volume studies.

\section{ACKNOWLEDGMENTS}

L.D. D. kindly acknowledges the hospitality of the Theory Department at CERN, where part of this work has been carried out. L. D. D. is supported by STFC, Grant No. ST/L000458/1, and the Royal Society, Wolfson Research Merit Award, Grant No. WM140078. B. L. is supported by STFC (Grant No. ST/L000369/1). C. P. is supported by a Lundbeck Foundation fellowship and by the $\mathrm{CP}^{3}$-Origins Centre which is partially funded by the Danish National Research Foundation, Grant No. DNRF90. A. R. is supported by the Leverhulme Trust, Grant No. RPG2014-118, and STFC, Grant No. ST/L000350/1. The numerical computations have been carried out using resources from the DiRAC Blue Gene Q Shared Petaflop system, HPC Wales and the High Performance Computing Center (HPCC) Plymouth. The DiRAC Blue Gene Q Shared Petaflop system is operated by the Edinburgh Parallel Computing Centre on behalf of the STFC DiRAC HPC Facility. This equipment was funded by BIS National E-infrastructure Capital Grant No. ST/ K000411/1, STFC Capital Grant No. ST/H008845/1, and STFC DiRAC Operations Grants No. ST/K005804/1 and No. ST/K005790/1. DiRAC is part of the National E-Infrastructure. The HPC Wales infrastructure is supported by the ERDF through the WEFO, which is part of the Welsh Government. 
[1] S. Weinberg, Phys. Rev. D 13, 974 (1976).

[2] L. Susskind, Phys. Rev. D 20, 2619 (1979).

[3] E. Eichten and K. D. Lane, Phys. Lett. 90B, 125 (1980).

[4] J. OlsenLHC seminar, ATLAS and CMS physics results from Run 2, talks by Jim Olsen and Marumi Kado, CERN, 15 Dec. 2015; ATLAS-CONF-2015-081, Search for resonances decaying to photon pairs in $3.2 \mathrm{fb}^{-1}$ of $p p$ collisions at $\sqrt{s}=13 \mathrm{TeV}$ with the ATLAS detector.

[5] M. KadoLHC seminar, ATLAS and CMS physics results from Run 2, talks by Jim Olsen and Marumi Kado, CERN, 15 Dec. 2015; CMS PAS EXO-15-004, Search for new physics in high mass diphoton events in proton-proton collisions at $13 \mathrm{TeV}$.

[6] K. Yamawaki, M. Bando, and K.-i. Matumoto, Phys. Rev. Lett. 56, 1335 (1986).

[7] T. Appelquist and L. C. R. Wijewardhana, Phys. Rev. D 36, 568 (1987).

[8] D. K. Hong, S. D. Hsu, and F. Sannino, Phys. Lett. B 597, 89 (2004).

[9] D. D. Dietrich, F. Sannino, and K. Tuominen, Phys. Rev. D 72, 055001 (2005).

[10] M. A. Luty and T. Okui, J. High Energy Phys. 09 (2006) 070.

[11] M. A. Luty, J. High Energy Phys. 04 (2009) 050.

[12] R. Contino, Y. Nomura, and A. Pomarol, Nucl. Phys. B671, 148 (2003).

[13] K. Agashe, R. Contino, and A. Pomarol, Nucl. Phys. B719, 165 (2005).

[14] D. K. Hong and H.-U. Yee, Phys. Rev. D 74, 015011 (2006).

[15] J. Hirn, A. Martin, and V. Sanz, Phys. Rev. D 78, 075026 (2008).

[16] C. Nunez, I. Papadimitriou, and M. Piai, Int. J. Mod. Phys. A25, 2837 (2010).

[17] D. Elander, C. Nunez, and M. Piai, Phys. Lett. B 686, 64 (2010).

[18] L. Anguelova, Nucl. Phys. B843, 429 (2011).

[19] D. Elander, A. F. Faedo, C. Hoyos, D. Mateos, and M. Piai, J. High Energy Phys. 05 (2014) 003.

[20] T. Alho, N. Evans, and K. Tuominen, Phys. Rev. D 88, 105016 (2013).

[21] C. Hoyos, U. Kol, J. Sonnenschein, and S. Yankielowicz, J. High Energy Phys. 10 (2013) 181.

[22] A. F. Faedo, M. Piai, and D. Schofield, Nucl. Phys. B880, 504 (2014).

[23] N. Evans and M. Scott, Phys. Rev. D 90, 065025 (2014).

[24] J. Erdmenger, N. Evans, and M. Scott, Phys. Rev. D 91, 085004 (2015).

[25] T. Appelquist, G. T. Fleming, and E. T. Neil, Phys. Rev. Lett. 100, 171607 (2008).

[26] A. J. Hietanen, J. Rantaharju, K. Rummukainen, and K. Tuominen, J. High Energy Phys. 05 (2009) 025.

[27] A. J. Hietanen, K. Rummukainen, and K. Tuominen, Phys. Rev. D 80, 094504 (2009).

[28] T. Karavirta, J. Rantaharju, K. Rummukainen, and K. Tuominen, J. High Energy Phys. 05 (2012) 003.

[29] Y. Shamir, B. Svetitsky, and T. DeGrand, Phys. Rev. D 78, 031502 (2008).

[30] A. Deuzeman, M. P. Lombardo, and E. Pallante, Phys. Rev. D 82, 074503 (2010).

[31] L. Del Debbio, B. Lucini, A. Patella, C. Pica, and A. Rago, Phys. Rev. D 82, 014510 (2010).
[32] L. Del Debbio and R. Zwicky, Phys. Rev. D 82, 014502 (2010).

[33] L. Del Debbio and R. Zwicky, Phys. Lett. B 700, 217 (2011).

[34] L. Del Debbio and R. Zwicky, Phys. Rev. D 89, 014503 (2014).

[35] F. Bursa, L. Del Debbio, L. Keegan, C. Pica, and T. Pickup, Phys. Rev. D 81, 014505 (2010).

[36] S. Catterall, L. Del Debbio, J. Giedt, and L. Keegan, Phys. Rev. D 85, 094501 (2012).

[37] T. DeGrand, Phys. Rev. D 84, 116901 (2011).

[38] A. Patella, Phys. Rev. D 86, 025006 (2012).

[39] K.-I. Ishikawa, Y. Iwasaki, Y. Nakayama, and T. Yoshie, Phys. Rev. D 87, 071503 (2013).

[40] K. I. Ishikawa, Y. Iwasaki, Y. Nakayama, and Y. Yoshie, Phys. Lett. B 748, 289 (2015).

[41] Y. Aoki, T. Aoyama, M. Kurachi, T. Maskawa, K.-i. Nagai, H. Ohki, A. Shibata, K. Yamawaki, and T. Yamazaki, Phys. Rev. D 86, 054506 (2012).

[42] Y. Aoki, T. Aoyama, M. Kurachi, T. Maskawa, K.-i. Nagai, H. Ohki, E. Rinaldi, A. Shibata, K. Yamawaki, and T. Yamazaki, Phys. Rev. Lett. 111, 162001 (2013).

[43] Y. Aoki et al. (LatKMI), Phys. Rev. D 89, 111502 (2014).

[44] Z. Fodor, K. Holland, J. Kuti, D. Nogradi, and C. Schroeder, Phys. Lett. B 703, 348 (2011).

[45] Z. Fodor, K. Holland, J. Kuti, D. Nogradi, C. Schroeder, and C. H. Wong, Phys. Lett. B 718, 657 (2012).

[46] T. Appelquist, G. Fleming, M. Lin, E. Neil, and D. Schaich, Phys. Rev. D 84, 054501 (2011).

[47] T. Appelquist et al. (LSD), Phys. Rev. D 90, 114502 (2014).

[48] F. Sannino and K. Tuominen, Phys. Rev. D 71, 051901 (2005).

[49] L. Del Debbio, B. Lucini, A. Patella, C. Pica, and A. Rago, Phys. Rev. D 82, 014509 (2010).

[50] L. Del Debbio, B. Lucini, A. Patella, C. Pica, and A. Rago, Phys. Rev. D 80, 074507 (2009).

[51] T. Karavirta, A. Mykkanen, J. Rantaharju, K. Rummukainen, and K. Tuominen, J. High Energy Phys. 06 (2011) 061.

[52] J. Rantaharju, T. Rantalaiho, K. Rummukainen, and K. Tuominen, arXiv:1510.03335.

[53] T. DeGrand, Phys. Rev. D 80, 114507 (2009).

[54] L. Del Debbio and R. Zwicky, Phys. Lett. B 734, 107 (2014).

[55] F. Bursa, L. Del Debbio, D. Henty, E. Kerrane, B. Lucini, A. Patella, C. Pica, T. Pickup, and A. Rago, Phys. Rev. D 84, 034506 (2011).

[56] L. Del Debbio, A. Patella, and C. Pica, Phys. Rev. D 81, 094503 (2010).

[57] G. 't Hooft, Nucl. Phys. B153, 141 (1979).

[58] M. Luscher and S. Schaefer, Comput. Phys. Commun. 184, 519 (2013).

[59] B. Lucini, A. Rago, and E. Rinaldi, J. High Energy Phys. 08 (2010) 119.

[60] M. Luscher and S. Schaefer, J. High Energy Phys. 07 (2011) 036.

[61] M. Bruno, P. Korcyl, T. Korzec, S. Lottini, and S. Schaefer, Proc. Sci., LATTICE2014 (2014) 089 [arXiv:1411.5207].

[62] M. Bruno et al., J. High Energy Phys. 02 (2015) 043.

[63] M. Luscher, Commun. Math. Phys. 104, 177 (1986).

[64] Y. Koma and M. Koma, Nucl. Phys. B713, 575 (2005). 
[65] A. Patella, L. Del Debbio, B. Lucini, C. Pica, and A. Rago, Proc. Sci., LATTICE2011 (2011) 084 [arXiv:1111.4672].

[66] N. Madras and A. D. Sokal, J. Stat. Phys. 50, 109 (1988).

[67] U. Wolff (ALPHA), Comput. Phys. Commun. 156, 143 (2004); 176, 383(E) (2007).

[68] M. Luscher, Comput. Phys. Commun. 165, 199 (2005).
[69] T. DeGrand and A. Hasenfratz, Phys. Rev. D 80, 034506 (2009).

[70] It is worth recalling that scaling is expected in the limits of large size and small mass. Observable-dependent deviations from scaling should be expected at small volumes or large masses. 